\title{
A modified device for removing large regurgitation volume during tracheal intubation
}

\author{
Kuo-Chuan Hung, MD $\cdot$ Shao-Wei Hsieh, MD
}

Received: 28 January 2016/Revised: 2 February 2016/Accepted: 8 February 2016/Published online: 17 February 2016

(C) Canadian Anesthesiologists' Society 2016

\section{To the Editor}

Gastric regurgitation occasionally occurs during tracheal intubation, sometimes involving a large amount of gastric contents, including significant particulate matter. The regurgitated material quickly fills the posterior pharynx, obscuring the view of the glottis and leading to significant pulmonary aspiration. Immediate, effective removal of this regurgitated material is important to maintain a clear airway and avoid the consequences of aspiration. Food particles, however, may easily obstruct standard suction cannulas, which have relatively small ports, making it difficult to remove a large regurgitated volume. Previous reports have demonstrated that an endotracheal tube (ETT) could function as a suction device to clear the airway. ${ }^{1,2}$ However, additional equipment is needed to connect the ETT to a suction source, and it may not be immediately available in the operating room. We herein describe a modified suction technique that allows expeditious clearance of regurgitated vomitus during tracheal intubation.

A suction device is assembled as shown in the Figure. The Opti-Port ${ }^{\mathrm{TM}}$, a right-angle, double-swivel connector
(Figure A) of a double-lumen tube (Broncho-Cath; Mallinckrodt, Athlone, Ireland), is attached to the ETT. The side-arm plastic tube of the swivel connector is then attached to the suction system (Figure B). This assembly enables the ETT to function as a suction route when the regurgitation volume is large. In the absence of regurgitation, the ETT can then be passed through the glottis and used conventionally. The operator's finger is used to occlude the bronchoscope port on the swivel connector to control the suctioning. Once it is used for suctioning, the ETT should be replaced with a new ETT. In addition, if needed, a stylet or other bougie can also be inserted through the bronchoscope port into the ETT lumen, allowing airway manipulation in the usual fashion (Figure B). It can be removed quickly if there is a large amount of regurgitated material.

This method readily permits suctioning of large-volume, particulate regurgitation during tracheal intubation. As the Opti-Port $^{\mathrm{TM}}$ swivel connector is usually nearby in the operating room, this easy-to-assemble, modified suction device may be helpful for managing patients at high risk of gastric regurgitation.
K.-C. Hung, MD $(\bowtie) \cdot$ S.-W. Hsieh, MD

Department of Anesthesiology, E-DA Hospital, I-Shou

University, Kaohsiung, Taiwan, ROC

e-mail: ed102605@gmail.com 


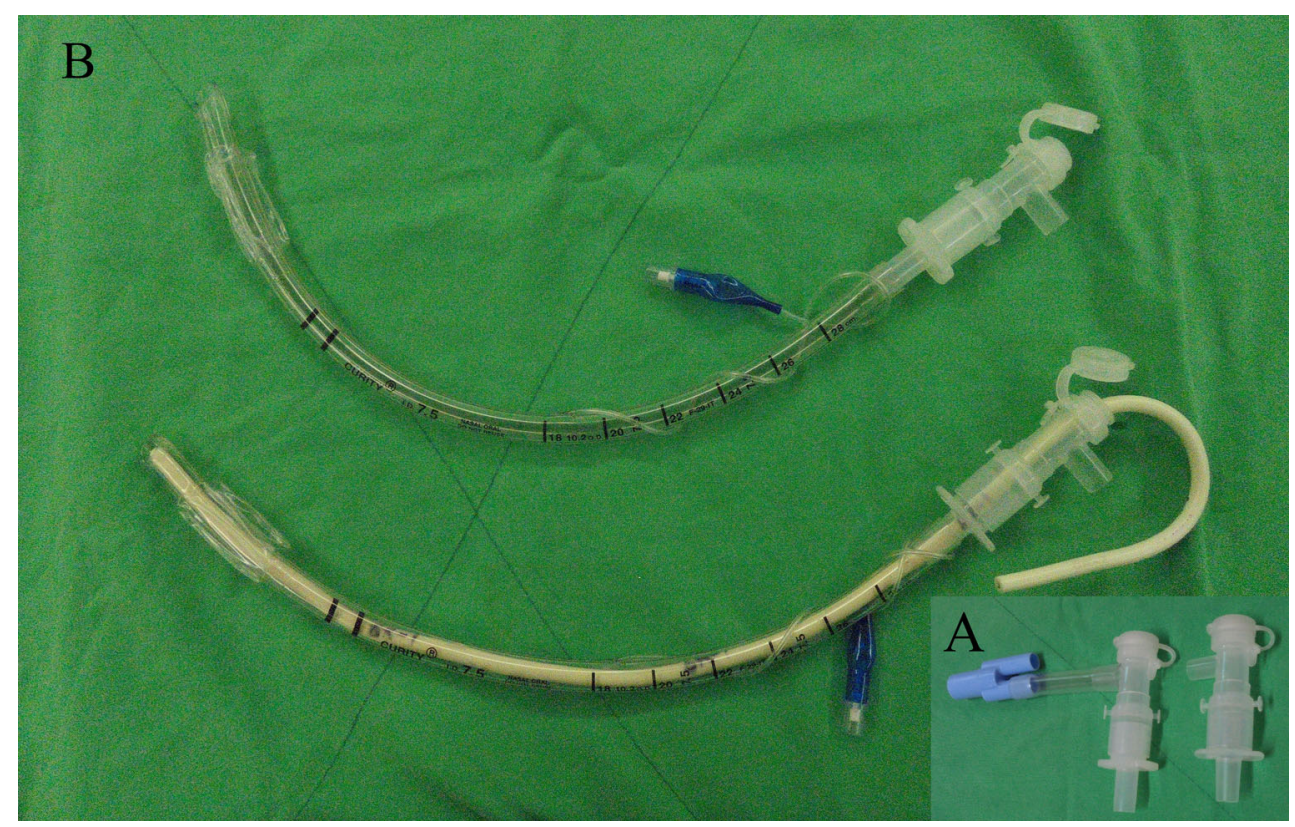

Figure A modified device for high-capacity suction, the OptiPort $^{\mathrm{TM}}$, a right-angle, double-swivel connector (Figure A) from a double-lumen tube, is attached to the endotracheal tube (ETT). The side-arm plastic tube of the swivel connector is then attached to the suction system (upper ETT) (Figure B). This assembly enables the ETT to function also as a high-capacity suction device. The operator

\section{Conflicts of interest None declared.}

Funding No external funding.

\section{References}

1. Bodenham AR. Removal of obstructing blood clot from the lower airway: an alternative suction technique. Anaesthesia 2002; 57: 40-3. can then control the suction system by occluding the bronchoscope port on the swivel connector with a finger. Also, a stylet can be inserted through the bronchoscope port into the ETT lumen (lower ETT), thereby allowing airway manipulation in the usual fashion. It can be quickly removed if there is a large volume of gastric regurgitation

2. Ruben H, Hansen E, MacNaughton FI. High capacity suction technique. A method of reducing the aspiration hazard during induction. Anaesthesia 1979; 34: 349-51. 